\title{
Cultura Organizacional y Gestión de la Calidad en una Empresa del Estado venezolano
}

\author{
Salcedo, Irma* \\ Romero, Juan José ${ }^{\star \star}$
}

\section{Resumen}

Este trabajo tiene como finalidad identificar los factores de la cultura organizacional que facilitan la implantación del sistema de gestión de la calidad en una empresa básica del Estado venezolano. Para ello se realizó una investigación de campo, con el propósito de analizar las percepciones de los directivos que conforman el nivel gerencial, en torno a tres variables a saber: La Filosofía de Gestión, el Estilo Gerencial y la Cultura Organizacional. En la Filosofía de Gestión se exploró el nivel de identificación de los gerentes con la misión, la estructura organizativa y los objetivos de la empresa. En el estilo gerencial se determinó el comportamiento de sus indicadores más relevantes como son; el liderazgo, la toma de decisiones, la solución de problemas y la asignación de tareas. Y en el abordaje de la cultura como tal, se trabajaron las representaciones axiológicas en torno a los valores, los principios y antivalores del nivel estratégico de la organización. Como resultado de esta investigación, se determinaron las cualidades humanas que deben internalizar los principales actores de la cultura organizacional, para alcanzar las exigencias que plantea el camino hacia la calidad, en los términos en que ha sido normada por estándares internacionales que definen las características óptimas del proceso productivo. En este sentido, se concluye que el trabajo en equipo, el aplanamiento de estructura, la reducción de los niveles jerárquicos y el mejoramiento de la comunicación interna, son cualidades que requiere una acción gerencial orientada a la gestión de la calidad de la empresa estudiada.

Palabras clave: Cultura organizacional, filosofía de gestión, gestión de la calidad, estilo gerencial.

Recibido: 05-05-09. Aceptado: 06-01-17

* Socióloga, Master en Gerencia de Recursos Humanos. CVG Venalum. Coordinadora de de entrenamiento y desarrollo. E-mail: irmasalcedo58@yahoo.com

** Sociólogo, Master en Gestión Universitaria, Doctor en Ciencias Económicas. Universidad Nacional Experimental de Guayana. Profesor-Investigador. E-mail: juanjose-r-s@cantv.net 


\title{
Cultural Organization and Quality Management in a Venezuelan State Company
}

\begin{abstract}
The objective of this paper was to identify the cultural, organizational factors that facilitate the implementation of a quality management system in a basic economic activity of the Venezuelan State. In order to do this a field study was undertaken for the purpose of analyzing the perceptions of the directors who participate at the management level in relation to three variables: management philosophy, management style, and organizational culture. The philosophy of management explores the level of manager identification with the mission, the organizational structure, and the objectives of the firm. In management style, the behaviour of the more relevant indicators such as leadership, decision-making, problem solving and the assignment of tasks are studied. In organizational culture, axiological representations as to values, principles and anti-values in the strategic level of organization are studied. As a result of this research, human qualities that should internalize the principle actors of the cultural organization, in order to reach the requirements proposed were determined. in order to reach quality based on international norms. In this sense, it was concluded that teamwork, the levelling of the structure, the reduction of hierarchic levels, and the improvement of internal communications were areas that required managerial action oriented towards quality management in the company under study.
\end{abstract}

Key words: Organizational culture, management philosophy, quality management, management style.

\section{Introducción}

Toda Organización que se mantenga proclive a cambios permanentes, en los procesos de mejoramientos continuos y en la consolidación de una filosofía de gestión que apunte hacia la excelencia, debe plantearse como una cuestión de primer orden, la revisión de lo que se llama la "cultura organizacional". Esta es en esencia la dimensión espiritual y conductual, que rige al agrupamiento humano en la empresa, donde coexisten sus miembros en una interrelación compleja. Hoy en día, las empresas de producción y servicios tratan de incorporar un patrón de comportamiento general, a través de la transmisión de creencias y valores comunes a sus miembros, de alguna manera contemplados en su misión institucional. Lo que explícitamente se busca es incorporar un espíritu de cuerpo, que contribuya a elevar la cohesión interna y a profundizar el sentido de pertenencia.

De allí ha resultado la gran relevancia del análisis de la cultura en la organización, la cual puede llegar a facilitar o dificultar los cambios. Tanto los valores como las creencias, contenidas generalmente en la filosofía de gestión, orientan a los trabajadores y les da identidad, los integra o los fracciona como miembros de una estructura productiva.

La empresa estudiada está en proceso de cambios importantes, entre los cuales se pueden destacar la adecuación a las exigencias normativas de un sistema de gestión de la calidad, que ha sido 
desarrollado en los lineamientos de la Internacional Standard Organization (ISO). Esta es una organización mundial ubicada en Ginebra, Suiza, fue creada en 1948 y está conformada por 140 países, entre los que se encuentra Venezuela. El objetivo de la Internacional Standard Asociation es definir los estándares en busca de un consenso en torno a la calidad de las actividades productivas, que abarcan el diseño de los procesos y la aplicación de normas con especificaciones técnicas. En Venezuela el organismo evaluador y acreditador es el Fondo para la Normalización y Certificación de la Calidad (FONDONORMA), que es una institución sin fines de lucro creada para otorgar las certificaciones de la Comisión Venezolana para Normas Industriales COVENIN. La norma ISO 9001 (FONDONORMA, 2000) se refiere a la gestión y aseguramiento de la calidad, a través de un sistema aplicable al diseño, desarrollo, fabricación, instalación y servicio posventa que se desarrolla en la empresa.

En concreto lo que se planteó la empresa fue lograr esta certificación, que es reconocida a nivel internacional, para el avance de los niveles de competitividad. Previamente a este proceso de normalización se había reforzado el modelo de gestión para alcanzar la excelencia en todos sus procesos bajo un enfoque de mejoramiento continuo y la implantación de un sistema de información automatizado, que utiliza como herramienta un prototipo de tecnología avanzada, conocido como SAP R/3.

El propósito de este artículo es comprobar que la cultura organizacional constituye un elemento estratégico para la consolidación del Modelo de Gestión
Empresarial. Por ello se asumió la perspectiva de la Sociología del Trabajo, para considerar las percepciones y representaciones colectivas de la totalidad del personal del nivel gerencial de la empresa.

Esta empresa se caracteriza por mantener permanentes cambios que apuntan hacia la consecución de resultados altamente productivos y el incremento en sus niveles de rentabilidad, frente a una realidad compleja, caracterizada por la presencia de un mercado global que demanda competitividad.

Esta demanda implica por supuesto, la revisión de los procesos internos, tanto a nivel de la tecnología dura, para verificar si los procesos tecnológicos son los más adecuados, si se cuenta con los equipos y maquinarias necesarias para producir eficientemente, como a nivel de la tecnología blanda, con la caracterización de la fuerza laboral con que cuenta, identificando sus fortalezas y debilidades, su estructura organizativa, su nivel de flexibilidad y autonomía. En este sentido, se trata de determinar si el recurso humano está alineado con los principios y valores, que están declarados y son resultado de una visión compartida por todos los miembros de la organización.

Si la dirección es hacia la competitividad, indudablemente no podemos obviar que la cultura organizacional, tiene un impacto importante en la conducta de sus trabajadores, porque puede incrementar o no la motivación de los mismos y los resultados de la organización. En muchos casos se manejan cambios de tecnologías, reestructuraciones, modificaciones de procesos, sin pensar en la necesidad de revisar lo que hace que la gente haga las cosas, más allá de la re- 
Cultura Organizacional y Gestión de la Calidad en una Empresa del Estado venezolano Salcedo, Irma y Romero, Juan José

muneración por la contraprestación de sus servicios.

Existen muchos autores que han trabajado acerca de la cultura organizacional y coinciden en definir que es el conjunto de entendimientos importantes que los miembros de una comunidad tienen en común. Es un sistema de valores y creencias compartidos, donde la gente, la estructura organizacional, los procesos de toma de decisiones y los sistemas de control interactúan para producir normas de comportamiento. Una determinada cultura puede contribuir al éxito de las organizaciones a largo plazo, al guiar el comportamiento y dar significado a las actividades. Los valores compartidos facilitan también la motivación, la cooperación y el compromiso.

Granell y Garaway (1997) en su libro "Éxito Gerencial y Cultura" señalaron hace algunos años, que las empresas estaban apenas empezando a descubrir que cambiar no es fácil y que los cambios estratégicos debían estar apoyados por los valores, actitudes y conductas de su gente. Sostiene además que fueron muy pocas las organizaciones que vieron en la cultura un problema importante y solamente aquellas que estaban haciendo un intento genuino de analizar su estilo gerencial, explorar el sentir de su gente, observar sus conductas y entender sus necesidades y expectativas -es decir, su cultura- estaban logrando resultados y progresos en sus programas de cambio.

Como caso de estudio tenemos a una empresa básica, que por razones estratégicas del estado venezolano, se decidió no identificar plenamente en la presentación de este artículo, debido a la información interna que aquí se maneja. A ella nos referiremos como una empresa procesadora de materia prima, que fue creada en 1978, y desde el año 1995 viene realizando esfuerzos en la consolidación de un Sistema de Calidad para la obtención de la Certificación ISO-9000, cuyos puntos focales son la satisfacción del cliente y el mejoramiento continuo de los procesos. Por otra parte, a partir del año 2000 , se dio inicio a otro proceso de implantación de un Nuevo Modelo "Excelencia de Gestión”, acompañado del SAP $R / 3$. Este sistema consiste en el diseño de una plataforma automatizada de los procesos administrativos y de manejo de materiales, de la gestión de procura y de reserva, que permitirá disponer de información en tiempo real a nivel de costos, presupuestos, inventarios y despachos, para facilitar el control y la toma de decisiones.

Este trabajo se orienta hacia la consolidación de un modelo de gestión empresarial, que contempla entre uno de sus factores el mantenimiento de un sistema de calidad, para lo cual es necesario que la cultura organizacional se convierta en un elemento estratégico, que debe ser conocida, validada y ubicada como el pilar fundamental para la implantación de este modelo, y a su vez coadyuve a la existencia de una empresa sana, robusta y altamente competitiva.

La investigación se desarrolló de acuerdo a una lógica hipotético-deductiva que permitió en primer lugar, la caracterización del objeto de estudio; en segundo lugar, la formulación de una hipótesis de trabajo, y, en tercer lugar, la contextualización de las variables más importantes y el estudio de los indicadores como elementos concretos de investiga- 
ción. Se planteó trabajar sobre la siguiente hipótesis: "La cultura organizacional influye en la implantación de un sistema de gestión de calidad". La operacionalización de esta hipótesis conlleva a una descripción de las variables y los indicadores tal como sigue: variable cultura organizacional, para la cual identificamos los siguientes indicadores: valores, antivalores y principios. Variable: estilo gerencial, con los siguientes indicadores: liderazgo, responsabilidad directiva, toma de decisiones, asignación de tareas y manejo de conflictos. Variable calidad, de la cual da cuenta: La misión, la estructura organizativa y los objetivos estratégicos, es decir la filosofía de Gestión.

La población objeto de estudio la conformaron todos los gerentes de la empresa que asciende a un total de dieciocho, a quienes se les realizó una entrevista durante los meses de agosto y septiembre del 2001. Es conveniente enfatizar que esta empresa, perteneciente a la Corporación Venezolana de Guayana (CVG), estuvo, a mediados de los años 1990, dentro de los planes de privatización, que formaron parte de los ajustes de la política económica, que el gobierno de la época trató de imponer por vía de decreto, como una "alternativa segura" para incrementar los niveles de rentabilidad y productividad, relegando el rol del estado en la conducción del desarrollo de la industria básica.

Este enfoque respondió a una lógica neoliberal, cuya pretensión más importante era la de privatizar esta empresa pública, lo cual evidenciaba una falta de compromiso con los intereses nacionales. A pesar de que el plan privatizador se trató de imponer desde el organismo rector del desarrollo regional, como es la Corporación Venezolana de Guayana (CVG), no se pudo concretar, ya que fue desechada por la nueva dirección institucional del país, que a partir del año 2000 retomó la conducción de las empresas básicas, como espacios públicos que definitivamente debían permanecer en manos del estado. Durante ese año, la Corporación Venezolana de Guayana (CVG), dirigida por una nueva gerencia, se replanteó el patrón del desarrollo industrial, lo cual requirió transformar la empresa con un nuevo modelo de gestión, para promover la participación de todos los trabajadores, llamados a incrementar el mejoramiento de la producción, con una visión estratégica y un liderazgo comprometido con el desarrollo endógeno, que exigía alinear la cultura organizacional con el nuevo modelo de gestión pública.

En el caso concreto de la empresa estudiada, se orientó una transformación que tuvo entre sus objetivos lograr la obtención de la certificación ISO 9001, dentro de lo cual se consideró como un asunto de gran importancia investigar como era visto el estilo gerencial, por sus propios actores en la empresa. En ese orden, se seleccionó la nómina de los gerentes por considerar que en este nivel la cultura organizacional puede ser afianzada y modelada para el resto de la organización, de allí que se trabajó con todos los gerentes, tanto del área operativa como de las áreas administrativas, incluyendo al presidente. La investigación realizada se planteó recabar la información para describir las representaciones de los gerentes, acerca de la cultura organizacional y la autoimagen que ellos tienen de su estilo gerencial. Por ello, no se profun- 
Cultura Organizacional y Gestión de la Calidad en una Empresa del Estado venezolano Salcedo, Irma y Romero, Juan José

dizó acerca de las implicaciones ideológicas, subyacentes en los datos obtenidos de los entrevistados. Esto permitió describir la autocalificación de estos actores, sin confrontarlo con las opiniones de otros niveles de la organización.

Al respecto, se considera importante ratificar que esta descripción se ubica como una aproximación a la cultura organizacional y el estilo gerencial, vistos desde la perspectiva de la gerencia de la empresa para el periodo investigado, por cuanto interesaba captar su predisposición hacia la aplicación de las nuevas políticas de transformación para el reposicionamiento de la dirección pública, en el desarrollo de esa industria.

\section{Aspectos Teóricos}

La premisa fundamental de la gestión de la calidad, la encontramos en Cantú (2001), quién la expresa en la búsqueda intencionada de la responsabilidad de los directivos para la lograr la excelencia, a través del mejoramiento permanente de los procesos que están bajo su responsabilidad. Según los lineamientos de Instituto Venezolano de Planificación (IVEPLAN, 1991), con la aplicación de este enfoque se intenta provocar una revolución, a través de un cambio de la filosofía de gestión hacia tres direcciones: A) La planificación de la calidad en función de las necesidades del cliente. B) El control de calidad u observación estadística de los procesos. C) La mejora de la calidad a partir del diseño de proyectos, que permitan enfrentar los problemas detectados.

Es conveniente señalar que en la filosofía de gestión de la empresa, el enfo- que de gestión de la calidad constituye un complemento del enfoque estratégico corporativo, con el cual se identifica el sistema de planificación del Instituto Venezolano de Planificación (IVEPLAN, 1993). La finalidad de este enfoque es, por una parte, construir la viabilidad de la organización, a través de una estructura que le permita adaptarse y responder a los cambios del ambiente interno y externo, y, por otro lado, lograr la eficiencia con la integración de los subsistemas técnico, psicosocial y el administrativo. Este enfoque también se plantea, operacionalizar los programas, a través del desarrollo coherente de las actividades que permiten que se concrete el plan estratégico corporativo. En el análisis de la organización y funcionamiento de la empresa estudiada, se observa una integración de los niveles estratégico, táctico y operativo que evidencia una visión compartida en la planificación, a través de la unidad de propósitos y compatibilidad de criterios técnicos, entre las instancias que participan en la gestión. Esta situación facilita la implantación del enfoque de gestión de la calidad.

La cultura organizacional es uno de los componentes de la acción gerencial que más influye en las empresas, y aun cuando esta afirmación constituye un lugar común, es necesario tenerla como punto de partida, sobre todo en aquellas empresas que buscan la excelencia. Por ello, se le considera como una de las ventajas comparativas de las organizaciones y la piedra angular del cambio y del mejoramiento continuo. La cultura ha resultado un factor clave, no solo para la gestión de recursos humanos, sino también para el avance de la tecnología. 
Algunos especialistas han fundamentado las teorías existente sobre el tema, como por ejemplo Robbins (1991), quien en la búsqueda de una explicación a los procesos sociales que se desarrollan en las organizaciones, señala que los trabajadores despliegan sus acciones en una relación de alta complejidad, con concepciones aprendidas, que muchas veces están en contradicción con la imagen de la empresa. De allí se considera que los valores culturales son indispensables para la organización y deben promoverse desde dentro de la empresa y estar incorporados en los instrumentos de planificación, tales como la visión, los objetivos estratégicos y la estructura organizativa, para que la organización funcione como sistema dinámico. Esto contribuye con la viabilidad del negocio al que se dedica en el marco de la globalización y competitividad. Por esta razón, uno de los asuntos que plantea el enfoque cultural de la organización es la calidad del liderazgo para conducir el cambio, relacionado con el manejo del poder, el rol, las tareas y las personas. Pero en general, se acepta que los valores, las creencias y los principios constituyen el verdadero sustento de un modelo gerencial, por lo que resulta muy complejo el análisis de la cultura de una manera objetiva, ya que ésta se expresa en percepciones manifiestas de los sujetos, a través del lenguaje, las normas, y las expresiones de sus líderes.

Resulta determinante conocer la cultura y rechazar las subculturas impregnadas de antivalores, por cuanto esto se constituye como una especie de discurso invisible que invade a toda la organización, mediatizando tanto a las per- sonas, como a sus espacios de actuación, e impidiéndoles la comprensión del funcionamiento de la organización: "Los efectos del mal conocimiento de la cultura son tristemente obvios en el ámbito internacional. El mal conocimiento de la cultura puede ser causa de guerras y del hundimiento de sociedades, como cuando la presencia de subculturas sólidas provoca que la cultura principal pierda su capacidad centralizadora y de integración" (Schein, 1988:47).

Una subcultura se asume como un conjunto de antivalores que adquieren cierta corporalidad, para oponerse a la cultura central y generar desequilibrio sistémico, por un lado, en el subsistema actitudinal, que siguiendo a Guédez (1996), abarca los comportamientos, sentimientos, relaciones, participación, entre otros. $\mathrm{Y}$, por otro lado, en el subsistema filosófico que se refiere a la misión, visión y valores de la organización.

Dentro de los enfoques de cultura hay una coincidencia al considerar entre sus factores; a los valores, antivalores y los principios, al lado de otros elementos que también la condicionan, como son el liderazgo, la responsabilidad directiva, la toma de decisiones, la delegación de tareas y el manejo de conflictos.

El análisis teórico de la cultura organizacional, encuentra entre sus principales especialistas a Ouichi (1982) quien señala que la cultura organizacional es una serie de símbolos, ceremonias y mitos que comunican al personal de la empresa los valores y creencias más arraigadas dentro de la organización.

También se encuentra Schein (1988), quien llama cultura a un modelo de presunciones básicas inventadas, 
Cultura Organizacional y Gestión de la Calidad en una Empresa del Estado venezolano Salcedo, Irma y Romero, Juan José

descubiertas o desarrolladas por un grupo dado al ir aprendiendo a enfrentarse con sus problemas de adaptación externa e integración interna, que hayan ejercido la suficiente influencia como para ser consideradas válidas $\mathrm{y}$, en consecuencia, ser enseñadas a los nuevos miembros como el modo correcto de percibir, pensar y sentir esos problemas.

Por otra parte, se destacan algunas definiciones, recopiladas por Cruz (2001), las cuales amplían el espectro de esta temática. Entre los autores manejados por Cruz tenemos, en primer lugar, a Petigrew, quien señala que la cultura de una empresa se manifiesta por los símbolos: lenguaje, mitos, ritos, entre otros, que son creados y difundidos por ciertos directivos para influir sobre el comportamiento de los miembros de la empresa. En segundo lugar, se encuentra Anzinzu, quien refiere que la cultura es un conjunto de principios y creencias básicas de una organización que son compartidas por sus miembros y que la diferencian de otras organizaciones. Luego tenemos a Pumpin, quien define a la cultura como el conjunto de normas y valores y de modos de pensar que marcan el comportamiento de los colaboradores a todos los niveles y que dan así su imagen a la empresa. También está Robbins, quien la denomina como la percepción que comparten todos los miembros de la organización, es decir el sistema de significados compartidos. Finalmente, tenemos a Mintzberg, quien señala que la cultura organizacional es la asunción por parte de los miembros de la organización de una peculiar forma de conducirse, que la diferencia de otras, junto a los objetos materiales de la organización.
Cruz (2001), después de haber estudiado estos autores define la cultura organizacional como una forma aprendida de hacer en la organización, que se comparte por sus miembros, consta de un sistema de valores y creencias básicas que se manifiesta en normas, actitudes, conductas, comportamientos, la manera de comunicarse, las relaciones interpersonales, el estilo de liderazgo, la historia compartida, el modo de dar cumplimiento a la misión y la materialización de la visión, en su interacción con el entorno, en un tiempo dado.

Según Granell (1997), la cultura organizacional es el conjunto de creencias, valores, supuestos y conductas compartidos y trasmitidos en una organización, que son adquiridos a lo largo del tiempo y que han sido exitosos para el logro de sus objetivos.

Para Romero (1996), la cultura organizacional es un conjunto de creencias desarrolladas por una organización en su proceso de interacción interna y adaptación externa, compartida por sus miembros, manifestada y trasmitida a través de diferentes expresiones: valores, ritos, normas, expectativas, actitudes, ceremonias, códigos de conducta, símbolos identificatorios e incluso formas arquitectónicas.

En este mismo orden, se plantea que la cultura es la expresión básica de la condición humana es decir, de la conciencia social a nivel de la organización y su propósito fundamental consiste en orientar a los ciudadanos que trabajan para que generen un sentido de pertenencia con la empresa, de la cual son la principal razón de ser. Se trata de la cotidianidad del trabajador en la organiza- 
ción en la que ha hecho historia y ha contribuido con el destino del colectivo.

Los enfoques anteriormente comentados, coadyuvan a revalorizar la concepción holística de la cultura como factor omnipresente en la organización, pues se considera que hoy no es posible operar una estructura funcional, ni diseñar políticas de productividad, sino se conocen los valores y creencias del colectivo empresarial, lo cual atañe tanto al sistema de dirección como a la división del trabajo y al entorno de la organización.

\section{Factores que intervienen en la cultura organizacional}

La cultura en la organización está afectada por las violentas transformaciones que vive el mundo laboral, con las innovaciones de los procesos y las estructuras, que son el resultado de la irrupción de un nuevo paradigma tecno-productivo, sustentado en la llamada sociedad del conocimiento: "Para entender más concretamente lo que significa un cambio de paradigma, vale la pena acercarse a ver la transformación que les toca vivir a los gerentes de hoy en su proceso de modernización. Todos estamos expuestos a los términos en boga: globalización, apertura, competitividad, sociedad del conocimiento [...] el cambio abarca todos los aspectos, disuelve todas las rutinas, cuestiona todos los hábitos y revoluciona cada uno de los tradicionales criterios de decisión" (Pérez, 1998:5).

Para comprender el cambio del modelo tecno-productivo, hay que contextualizar los elementos clave de la transformación, lo cual amerita un profundo replanteo de nuestra lectura del mun- do productivo que está impactando el escenario socio económico y el sistema jurídico-político, con sus consecuencias profundas en el campo educativo y cultural.

En otro orden de ideas, resulta fundamental el papel que juega el liderazgo en el manejo de la cultura, el cual es decisivo al considerarse, el ejercicio de tal liderazgo, como un arquetipo en la organización, que favorece cambios paulatinos, en la percepción, valoración y formas de enfrentar los problemas que se presentan en la organización.

Sin embargo Montero (1999), considera que paralelamente se puede desarrollar una estereotipación del liderazgo, que consiste en la exageración de los rasgos negativos que pueden originar posturas rígidas y prejuiciadas ante el cambio, es lo que se ha conceptualizado en esta investigación como los antivalores de la cultura organizacional. Dicho de otra manera, se trata de entender como los elementos culturales que sustentan el liderazgo pueden orientarse como factores inerciales que resisten el cambio. Esto puede comprometer, como fuerzas resistentes, a los actores en los distintos escenarios.

La alta dirección, por lo general, debe promover una filosofía y un estilo administrativo para cohesionar las conductas individuales en una visión compartida. De allí surge la concepción de la cultura como elemento de cohesión social o normativa que acopla a una organización. La cultura organizacional comporta lineamientos perdurables que condicionan la conducta individual para trasmitir sentimientos de identidad a los miembros de la organización, facilitar el compromiso, reforzar la estabilidad y re- 
Cultura Organizacional y Gestión de la Calidad en una Empresa del Estado venezolano Salcedo, Irma y Romero, Juan José

conocer premisas para la toma de decisiones.

El estudio de los valores o ideales sociales, y las creencias que los miembros de la organización llegan a compartir, se manifiestan en elementos simbólicos como mitos, rituales, historias, leyendas y un lenguaje especializado. Por lo general, estos elementos son esenciales en cualquier propuesta de transformación de la organización, pues al lado de los factores técnicos - productivos, es el hombre quien decide la direccionalidad del proceso. Ello reafirma la valoración que debemos tener de la cultura como una totalidad. La cultura organizacional comporta todo el pensamiento y la acción humana que se desarrolla en la organización, en el actual contexto de la mundialización.

A continuación se conceptualizan los principales factores de la cultura organizacional estudiados en la investigación de la empresa:

\subsection{Valores y antivalores}

El concepto de Valor tiene un sentido filosófico que proviene de la llamada Teoría de los Valores o Axiología. Es una dimensión intrínseca al ser humano, que hace referencia a la concepción del mundo y de la vida, se asume como cualidades ideales que carecen de corporalidad y se encuentran en la esfera del ser. Al respecto, es conveniente precisar lo siguiente: “...el valor depende de los sentimientos de agrado o desagrado, del hecho de ser o no deseado, de la subjetividad humana, individual o colectiva [...] los valores se presentan siempre polarmente, porque no son entidades indiferentes como las otras realidades. Al valor de la belleza se contrapone siempre el de la fealdad; al de la bondad, el de la maldad; al de lo santo, el de lo profano" (Ferrater, 1974: 426).

Los valores se encuentran vinculados al orden social, en la medida en que se asumen como comportamientos compartidos y legítimos que influyen en el proceso de socialización. Por eso se llega a afirmar que son elementos indispensables de la Cultura que activan el funcionamiento de las organizaciones humanas. Cantú (2001), señala que los valores son aquellas impresiones profundas, que se tienen de la forma en que se vive, sobre lo que se considera éticamente correcto o incorrecto, y que se lleva a la vida personal de forma congruente.

En esta investigación se asumió que los valores tienen una connotación positiva para la organización, por cuanto expresan los criterios que los trabajadores utilizan para interpretar la realidad organizacional, así como las relaciones interpersonales que establecen para lograr el éxito. Por ello, los valores organizacionales expresan un camino compartido por todos los trabajadores y directivos y su compromiso por el logro de las metas. De allí que se piensa que la búsqueda de la excelencia supone una sistematización e incorporación de los valores, para activar el comportamiento permanentemente sano en la empresa. Esto generalmente se logra a través de la misión y la visión, pero también en el modelaje de los directivos hacia el colectivo, lo cual hace que los valores se conviertan en refuerzos subjetivos de una gran motivación.

Para definir los antivalores, es necesario enfatizar el hecho de que sólo se refieren a una posición contradictoria de 
lo que se define como valor, pues resultan de una reflexión simplista de la realidad subjetiva, que se encuentra generalmente en el inconsciente del individuo. En los estudios psicosociales, se demuestra que los antivalores provienen de una imagen exageradamente simplificada de los rasgos negativos que se incorporan dentro de una cultura. Por ello, los antivalores aparecen como las conductas negativas que peyorativamente son utilizadas para excluir de la dinámica social a ciertos grupos:

"La simplificación de las características atribuidas, la exageración y amplificación de los rasgos negativos, así como la generalización, a la vez que atenúa, niega e ignora las características positivas, señalan hacia un proceso de estereotipación" (Montero, 1999:71).

De tal manera, en las Ciencias Sociales muy poco se ha trabajado la temática de los antivalores como conductas disfuncionales, sino más bien como representaciones del inconsciente colectivo, tal como lo apunta Montero (1999), cuando analiza la autoimagen del venezolano. Esto conlleva a una aplicación del concepto de antivalores, referido a los estereotipos que se producen en las organizaciones.

\subsection{Principios}

El término Principio, está asociado a la Filosofía Presocrática, en la búsqueda del "principio de todas las cosas" o "aquello de lo cual derivan todas las demás cosas". En este sentido, se asume que un Principio es una razón por la cual las cosas son lo que son. El principio es el punto de partida del movimiento de una cosa, o sea que el carácter común de todos los principios es ser la fuente de donde se deriva el ser y el conocimiento. Sin embargo, frecuentemente se presenta una definición reducida de los principios de cada ciencia, que operan como verdades absolutas y se conciben como proposiciones máximas para comprender la actividad humana. Siguiendo a Ostos (2000), en el caso de las Ciencias administrativas los Principios se presentan, como bases fundamentales sobre las cuales se apoyan las acciones, las reglas y las pautas que definen el modo como nos relacionamos con el entorno: los otros, la organización, la sociedad, el país, el planeta.

\subsection{Liderazgo}

El concepto del liderazgo está relacionado con un conjunto de componentes que se le atribuyen a una persona para conducir a un grupo u organización, lo cual requiere de conocimientos y habilidades y hasta de condiciones innatas, como el carisma. En la filosofía antigua de la China se observaba el liderazgo como: "La voluntad de controlar los sucesos, la comprensión necesaria para marcar un rumbo y el poder para llevar a cabo una tarea, utilizando cooperativamente las habilidades y las capacidades de otras personas" (Krause, 1998:23).

Ya hace dos mil quinientos años Sun Tzu, citado por Krause (1998), destacaba el papel del líder en el arte de la guerra, considerándolo fundamental para organizar los cambios de conductas sociales. Más tarde en la Sociología, se definió el liderazgo como el ejercicio de las élites para influir en las colectividades sociales; 
Cultura Organizacional y Gestión de la Calidad en una Empresa del Estado venezolano Salcedo, Irma y Romero, Juan José

es así como se han conceptualizado cuatro modelos correspondientes a las formas que adquiere el ejercicio de la autoridad a través del líder. Esto le asigna una connotación situacional, por lo que hoy se clasifica de la siguiente manera: liderazgo carismático: basado en condiciones innatas de simpatía y proyección personal, liderazgo democrático: basado en la autoridad del conocimiento y la promoción de la participación colectiva, liderazgo laissez faire: que se desarrolla a partir de las normas y costumbres y el Liderazgo Autocrático: que se ejerce por el poder que da el cargo, con esquemas impositivos sobre el colectivo.

\subsection{Responsabilidad directiva}

La responsabilidad directiva se refiere a la obligación de los Gerentes para dirigir el sistema productivo e integrar a la gente, en la consecución o logro de las metas. Se trata de un camino hacia la competitividad, que incluye además de los conocimientos técnicos, la posibilidad de transferir valor intelectual a las empresas generando capacidad para la creatividad e innovación de las personas. Por lo tanto, la responsabilidad de los directivos no sólo se refiere al esfuerzo por mejorar la calidad de los productos, con el uso adecuado de las tecnologías, sino a su preparación y formación constante para acelerar el cambio hacia una situación de justicia y equidad. Este indicador del estilo gerencial se trabajó como un componente indispensable en la presente investigación, y se revisó a partir de tres obligaciones que les corresponde asumir a los Gerentes, como son: Aseso- rar y apoyar al trabajador para un excelente desempeño laboral, controlar las funciones asignadas y evitar incumplimientos, y, resolver problemas mediante el trabajo en equipo. En otras palabras, la responsabilidad directiva contempla tres niveles: el apoyo al individuo, el control normativo y el trabajo en equipo.

\subsection{Toma de decisiones}

La toma de decisiones, se considera como un proceso mediante el cual se acuerda la ejecución de una acción que surge de la selección que se obtiene del análisis de un conjunto de opciones. Esta acción tendrá impacto en la organización y requerirá la intervención de las personas encargadas de llevarla a cabo. Por ello, destacamos el carácter colectivo que asumen las decisiones en el entorno laboral. En el caso de las decisiones que se toman en el nivel gerencial, frecuentemente se consideran tres factores fundamentales: La intuición y la experiencia, vinculadas a un tipo de decisión personal y espontánea, los objetivos y metas, que se asocian con un tipo de decisión institucional -estratégica, y, el análisis de los hechos obtenidos de la realidad, que se refiere a un tipo de decisión casuísticacoyuntural.

Por otro lado, el proceso de toma de decisiones involucra fundamentalmente al nivel estratégico de la organización, pero sin que ello implique que el resto de los niveles que la conforman estén exentos de decidir situaciones que competen a su ámbito de acción inmediata. 


\subsection{Asignación de tareas}

La asignación de tareas, es otro de los componentes del estilo gerencial, que implica una relación directa entre el supervisor y sus subordinados, para la ejecución de la división técnica del trabajo, a través de la distribución de las responsabilidades para el cumplimiento de los planes y metas establecidas, para lo cual se toman en cuenta tres factores básicos, a saber:

La valoración del tiempo estimado para su ejecución, la disposición de recursos y las capacidades y conocimientos para su realización efectiva. El ejercicio de asignar tareas, le permite al gerente evidenciar su proclividad a valorar a la persona, o al grupo. Esto se relaciona con dos tendencias fundamentales: Delegar y dividir responsabilidades hacia las personas, y, crear interdependencia y responsabilidad compartida en el grupo.

\subsection{Manejo de conflictos}

El manejo de conflictos exige, de parte de quienes interactúan en la relación laboral, una conciencia acerca de la diversidad de los intereses que están representados por quienes siendo diferentes, juegan roles complementarios para garantizar el funcionamiento de la organización. Sin embargo, el conflicto se hace inevitable en una situación concreta, cuando las partes se sienten en oposición acerca de los objetivos y las metas de la empresa. Davis y Newstrom (2000) describen dos tipos de conflicto: Interpersonal referidos a las contradicciones entre los roles de las personas, como evidencia de estas contradicciones, tenemos los conflictos que se producen entre el supervisor y el supervisado e intergrupal, que se producen por el sistema de lealtades y la competencia de los grupos por la distribución de los recursos y el poder en la empresa.

Entre las causas del conflicto se destacan: El cambio organizacional, choques de personalidad, diferencias en los sistemas de valores, amenazas al estatus, percepciones contradictorias y falta de confianza. Estas causas generan situaciones que pueden ser favorables para activar a la gente hacia el cambio con energía y creatividad y para buscar soluciones estables a problemas que permanecían ocultos. No obstante, pueden tener un efecto destructivo, en la medida en que son personalizadas las situaciones para buscar culpables y no para enfrentar los problemas, asumiendo un comportamiento cíclico en la organización. En cuanto a la resolución de los conflictos:

"Las estrategias más simples se basan en los contrastantes enfoques de cooperación o competencia [...] Cada una de ellas representan diferentes grados de interés en los resultados propios y en los de otra persona y tiene un efecto previsible" (Davis y Newstrom, 2000:343). Entre esas estrategias tenemos: Evitación: distanciamiento físico del conflicto. Suavizar: adaptación a los intereses de la otra parte. Forzamiento: uso de tácticas de poder para obtener beneficios. Negociación: búsqueda de un punto medio o disposición a renunciar a algo a cambio de obtener otra cosa, y. Confrontación: enfrentamiento directo del conflicto en busca de una solución mutuamente satisfactoria. Se considera que únicamente la 
Cultura Organizacional y Gestión de la Calidad en una Empresa del Estado venezolano Salcedo, Irma y Romero, Juan José

confrontación y la negociación resultan realmente efectivas para la resolución de los problemas, por cuanto implica actitudes creativas, desprejuiciadas y asertivas, que privilegian el interés colectivo de la organización para una convivencia armónica.

\subsection{Filosofía de gestión}

Para entender adecuadamente el papel de la filosofía de gestión en la empresa investigada, consideramos necesario, describir los siguientes elementos: La Misión, los objetivos corporativos y la estructura organizativa.

Cuando se refieren los términos filosofía de gestión de la organización, estamos hablando de los fundamentos doctrinarios de la gestión: "en tanto que en ella se establece su razón de ser, la dirección de su desempeño y conducción y modalidades de acción" (IVEPLAN, 1991:24). Es decir, el sistema de planificación debe determinar los propósitos y la razón de ser de la organización, esto es lo que corresponde definir al nivel estratégico en la misión de la Organización. Igualmente debe definir la dirección que debe seguir la gestión, a través de directrices, estrategias y objetivos estratégicos. Además deben determinarse las orientaciones que guiarán la toma de decisiones, lo cual permite definir las políticas generales de la organización.

La misión, como ya se ha sostenido, es el propósito o la razón de ser de la organización: "representa el faro que establece la dirección hacia el deber ser al que conducirá la organización" (IVEPLAN, 1991: 7). Entre los elementos a considerar para definir la misión tenemos:
Marco legal con sus instrumentos como la Constitución Nacional, las Leyes y reglamentos que regulan los parámetros de actuación de la organización.

Dinámica y tendencias de la realidad nacional e internacional, lo cual contempla los cambios económicos, sociales, políticos, tecnológicos, legales, entre otros.

Trayectoria institucional que contempla: la evolución histórica de la organización, la incidencia de situaciones sobre su orientación y la respuesta de la organización ante los cambios.

Presiones y expectativas sobre la organización: que son de carácter externo como las que ejercen organismos públicos, comunidad organizada y organismos internacionales. Y las de carácter interno del nivel directivo/gerencial, nivel funcional central y nivel zonal.

Los objetivos corporativos, se refieren a los propósitos o logros específicos que derivan de la misión de la organización, hacia los cuales se dirigirán todos los esfuerzos para alcanzar los productos, por lo cual la definición de ellos implica una intencionalidad, referida a lo que se desea obtener colectivamente. O mejor dicho, la direccionalidad de la organización es posible orientarla de una manera clara y realista mediante una secuencia de lineamientos operativos, que contemplan los alcances de la gestión a mediano plazo.

La estructura organizativa, consiste en la forma y configuración que adquieren un conjunto de niveles que se interrelacionan entre si para hacer funcionar la totalidad de la organización. Aunque cada nivel mantiene una autonomía relativa, siempre deben estar en articulación funcional. En términos del sistema de pla- 
nificación, en la estructura deben conformarse tres niveles fundamentales: Nivel de dirección suprema y superior, integrado por las máximas autoridades y su acción es estratégica porque se encarga de la filosofía de gestión. Nivel de gerencia media o coordinadora, integrado por las autoridades medias y su acción es táctico porque se encargan de los planes funcionales. Nivel operativo, integrado por el personal ejecutor y su acción consiste en operacionalizar los programas, proyectos y actividades.

\section{Resultados}

Los resultados que se presentan a continuación están relacionados con la percepción del personal gerencial con respecto a la filosofía de gestión, el estilo gerencial y la cultura organizacional, factores que podrían impactar en la empresa investigada para la futura implantación de un nuevo modelo de gestión, que tiene como premisa la búsqueda de la excelencia bajo el enfoque del sistema de gestión de la calidad de acuerdo a las exigencias de la Norma ISO 9001:2000.

\subsection{Filosofía de Gestión}

Con respecto a la filosofía de gestión los resultados son: En cuanto a la misión la opinión del personal gerencial resulta altamente favorable, pues 17 gerentes que representa el $94,5 \%$ conoce la misión de la organización, sólo un gerente manifestó desconocimiento, representando un 5,5\%, lo cual se explica por su reciente incorporación a la organización.

Los gerentes entrevistados evidencian un conocimiento aceptable de los niveles de la organización. Quince geren- tes demostraron conocimiento adecuado de la estructura de la empresa, lo cual representa el $83 \%$ del personal gerencial y sólo tres gerentes, que representan el $17 \%$ expresaron desconocimiento en este sentido. Lo cual puede obedecer a la situación de transición que se estaba operando para el momento en que se produjo la aplicación del instrumento ya que se estaba concretando la reestructuración organizativa que estaba en pleno proceso de aprobación, esto puede indicar un nivel de desactualización de la información, por parte de estos tres gerentes, quienes se mantenían con la idea de la anterior estructura.

Los dieciocho gerentes manifiestan un conocimiento exacto de los objetivos contenidos en el plan. Ello evidencia que este plan fue difundido adecuadamente por el ente responsable, resultando un elemento favorable para la ubicación de las metas establecidas y el uso racional de los recursos de que disponen, para el logro de la eficiencia.

\subsection{Estilo Gerencial}

En relación con el estilo gerencial, se investigó para conocer la práctica directiva de los gerentes y determinar los principales indicadores en que se fundamenta.

Para el indicador liderazgo se consideraron los atributos que, según la opinión personal del propio gerente, les resultan indispensables para dirigir al colectivo bajo su adscripción, tales como: Poder del cargo (liderazgo autocrático). Autoridad del conocimiento (liderazgo democrático). Normas y costumbres (liderazgo laissez faire). Simpatía por el personal (liderazgo carismático). 
Cultura Organizacional y Gestión de la Calidad en una Empresa del Estado venezolano Salcedo, Irma y Romero, Juan José

La percepción de quince gerentes (83\%) se ubica en el liderazgo democrático, es decir, al dirigir al colectivo utilizan fundamentalmente la autoridad que les da el conocimiento y sólo tres gerentes, el $17 \%$, se identifican con el liderazgo laissez faire, lo que expresa que utilizan las normas y costumbres para dirigir al colectivo. Ninguno de los encuestados asume los atributos del liderazgo autocrático, ni del liderazgo carismático.

En cuanto a responsabilidad directiva se evaluaron los siguientes aspectos: Asesoría y apoyo al trabajador, control de las funciones asignadas para evitar incumplimientos y solución de problemas mediante el trabajo en equipo

Las respuestas obtenidas por parte de los gerentes evidencian una dispersión en cuanto a los mecanismos que utilizan para cumplir su responsabilidad directiva. Encontrándose que nueve gerentes $(50 \%)$ prefieren el trabajo en equipo, seis gerentes que representan el 33\%, consideran que su responsabilidad debe centrarse en el apoyo al individuo y tres gerentes que representan el $17 \%$, asumen el control normativo como fundamento de su responsabilidad.

El indicador toma de decisiones se dimensionó en tres posiciones que soportan los mecanismos de la toma de decisiones, a saber: La intuición y la experiencia personal (personal-espontáneo), los objetivos y metas (institucional-estratégica), y el análisis de los hechos obtenidos de la realidad (casuística-coyuntural).

Las opiniones de los gerentes indican que los mecanismos por ellos utilizados, para el proceso de toma de decisiones, están equilibrados con la variante Institucional -estratégica, asumida por nueve de los gerentes que representan el $50 \%$ y la variante casuística- coyuntural asumida por los otros nueve gerentes que representan el $50 \%$ restante de la población, de lo cual se deriva que los gerentes descartan el criterio personal y espontáneo para apoyar sus decisiones. Los resultados reflejan que los gerentes realizan su proceso considerando los objetivos y metas de la organización, así como el análisis de los elementos que son dados por las situaciones que se presentan en la empresa.

Para el abordaje del indicador asignación de tareas se interrogó acerca de los criterios que se valoran para la asignación de las tareas a los trabajadores, referidos a: Delegar y dividir responsabilidades hacia las personas (valoración a la persona), y crear interdependencia y responsabilidad compartida en el grupo. (Valoración al grupo).

Esto dio como resultado una propensión hacia el trabajo grupal en las asignación de las tareas por parte de diez gerentes, que representan el $56 \%$ y preferencia por parte de ocho gerentes, es decir, el 44\%, hacia la valoración de la persona; lo cual indica cierto equilibrio en los dos criterios considerados para la asignación de tareas.

Finalmente el indicador manejo de conflictos se dimensionó a través de dos métodos excluyentes y antagónicos, para enfrentar los conflictos laborales, como son: La aplicación de mecanismos de represión y arbitraje externo (normativo-represivo), y la negociación y solución de problemas (negociación-consensual).

Como resultado se obtuvo una percepción absoluta del 100\% hacia el método de resolución de problemas a través 
de la negociación-consensual; por supuesto un rechazo hacia el método normativo-represivo, en el ámbito de las relaciones laborales con el personal bajo su adscripción.

\section{3. cultura organizacional}

El estudio de la cultura organizacional resulta determinante para ubicar el contenido axiológico de la percepción gerencial, que como se ha sostenido en el fundamento teórico de esta investigación, podría coadyuvar de manera significativa, con la predisposición necesaria para la implantación de un nuevo modelo de gestión, basado en una cultura de calidad.

Se presentaron para la consideración de los gerentes, los factores de la cultura organizacional, a través de un instrumento que se manejó con la finalidad de determinar las percepciones que estos gerentes presentaban como imagen de si mismo, en cuanto a valores, antivalores y principios.

Aquí no se trató de evaluar la práctica de los gerentes con respecto al ejercicio de los valores, antivalores y principios, por lo que debe quedar claro que esta puntuación no necesariamente corresponde a la acción gerencial vista desde la perspectiva crítica, lo cual ameritaría aplicar el instrumento de medición a los trabajadores para capturar una información que permita contrastar las respuestas de la acción gerencial desde estas dos perspectivas.

En relación con los valores se estableció una escala jerarquizada en orden de importancia, cada uno de ellos podía obtener un puntaje máximo de 360 , como producto de la multiplicación de las dieciocho opiniones, por veinte (mayor puntaje que podía asignarse). Los valores señalados en la encuesta, son los que típicamente se considerarían en cualquier cultura organizacional y responden a lo axiológico y personal de cada gerente. Los resultados obtenidos pueden verse en la Tabla 1.

Tabla 1

Puntuación asignada a los
Valores (Según la percepción
de los Gerentes de una empresa
del Estado Venezolano)

\begin{tabular}{|c|c|}
\hline Valores & Puntuación \\
\hline Responsabilidad & 326 Puntos - 90\% \\
\hline Honestidad & 305 Puntos $-\mathbf{8 1 . 7 \%}$ \\
\hline Respeto & 276 Puntos - $76.6 \%$ \\
\hline Disciplina & 265 Puntos - $73.6 \%$ \\
\hline Motivación al logro & 239 Puntos - $66,4 \%$ \\
\hline Lealtad & 231 Puntos $-64.7 \%$ \\
\hline Creatividad & 207 Puntos - 57.5\% \\
\hline Autoestima & 200 Puntos - 55.5\% \\
\hline $\begin{array}{l}\text { Disposición } \\
\text { al cambio }\end{array}$ & 192 Puntos - 53,3\% \\
\hline Desarrollo intelectual & 191 Puntos - 53\% \\
\hline Puntualidad & 180 Puntos - $50 \%$ \\
\hline Sinceridad & 171 Puntos - $47.5 \%$ \\
\hline Deseo de superación & 166 Puntos - $46.1 \%$ \\
\hline Orden & 145 Puntos - $\mathbf{4 0 . 3 \%}$ \\
\hline Gusto por el trabajo & 144 Puntos - $40 \%$ \\
\hline Bienestar familiar & 124 Puntos - $\mathbf{3 4 . 2 \%}$ \\
\hline Libertad & 123 Puntos $-34.2 \%$ \\
\hline Limpieza & 120 Puntos - $\mathbf{3 3 . 3} \%$ \\
\hline Generosidad & 78 Puntos - 21, $6 \%$ \\
\hline Amistad & 65 Puntos - $18 \%$ \\
\hline
\end{tabular}

Fuente: Elaboración propia a partir de la entrevista. 
Cultura Organizacional y Gestión de la Calidad en una Empresa del Estado venezolano Salcedo, Irma y Romero, Juan José

Se manejó el antivalor como una dimensión antagónica del componente axiológico que caracteriza la práctica gerencial, para lo cual se presentó una lista con cinco antivalores, para que los gerentes jerarquizaran de acuerdo a su opinión. Cada uno de ellos podía obtener un puntaje máximo de 90 , como resultado de la multiplicación de las dieciocho opiniones, por cinco (mayor puntaje que se podía asignar). Los resultados pueden verse en la Tabla 2.

Tabla 2

Puntuación asignada a los Antivalores (Según la percepción de los Gerentes de una empresa del Estado Venezolano)

\begin{tabular}{ll}
\hline Antivalores & Puntuación \\
\hline Deshonestidad & 85 Puntos - 94.4\% \\
Mentira & 62 Puntos - 68.8\% \\
Injusticia & 48 Puntos - 53.3\% \\
Agresividad & 42 Puntos - 46.6\% \\
Envidia & 33 Puntos - 36.6\% \\
\hline
\end{tabular}

Fuente: Elaboración propia a partir de la entrevista Puntuación máxima: 90

\section{Principios Gerenciales}

Se presentaron diez principios gerenciales, para ser jerarquizados en orden de importancia, del 1 al 10 y de acuerdo con las opiniones emitidas por los gerentes entrevistados. Cada uno de esos principios podía obtener un puntaje máximo de 180, como producto de la multiplicación de las dieciocho opiniones, por diez (mayor puntaje que podía asignarse). Los resultados se presentan en la Tabla 3 .
Tabla 3 Puntuación asignada a los principios (Según la percepción de los Gerentes de una empresa del Estado Venezolano)

\begin{tabular}{lc}
\hline Principios & \multicolumn{1}{c}{ Puntuación } \\
\hline Liderazgo & 166 Puntos - 92.2\% \\
Cooperación & 135 Puntos - 75\% \\
Reconocimiento & 118 Puntos - 65.5\% \\
Satisfacción al & 102 Puntos - 56.6\% \\
trabajador & \\
Comunicación & 94 Puntos - 52.2\% \\
Capacitación & 92 Puntos - 51.1\% \\
Creatividad & 80 Puntos - 44.4\% \\
Mejoramiento & \\
continuo & 79 Puntos - 43.8\% \\
Calidad de servicio & 65 Puntos - 36.1\% \\
Servicio al cliente & 59 Puntos - 32.7\% \\
\hline $\begin{array}{l}\text { Fuente: Elaboración propia a partir de la entrevista. } \\
\text { Puntuación máxima: } 180 .\end{array}$
\end{tabular}

\section{Análisis de los resultados}

En la presente investigación se determinó que los componentes de la cultura organizacional analizados, a partir de las percepciones de los gerentes, favorecen el proceso de implantación de un sistema de gestión de la calidad en la empresa, como uno de los objetivos a cumplir a corto y mediano plazo. En este marco, cabe señalar el alto grado de identidad que mantienen los directivos con la filosofía de gestión, con respecto a los tres componentes analizados, lo cual quiere decir, que los gerentes, desde el presidente hasta los gerentes de área, saben cuál es la direccionalidad de la empresa, cuál es su razón de ser, cuáles son sus 
objetivos, para el logro de esa misión y cuál es la estructura que la soporta.

La identidad y el sentido de pertenencia organizacional, que emanan de los gerentes, tienen relación directa con la inducción que han recibido estos directivos, por ser ésta una empresa del Estado, en la que se ha venido asumiendo un trabajo de equipo para lograr la cohesión alrededor de una visión compartida, lo cual ha estado acompañado de una difusión adecuada de los documentos, carteles y folletos donde se describe la filosofía de la empresa.

Otro de los aspectos que coadyuva con esta percepción que exteriorizan los gerentes con respecto a la filosofía, es la continuidad que se observa en los últimos años en los cargos directivos, basada en el reconocimiento de los méritos y el desarrollo de la carrera gerencial.

Con respecto a la percepción de los entrevistados acerca de la práctica directiva de los gerentes, en términos generales se encuadra dentro de un estilo proactivo, del liderazgo transformador, observándose una opinión de poca resistencia al cambio y una clara comprensión de la relación entre la cultura organizacional y la productividad. En este punto, es necesario señalar que a pesar de la obtención de respuestas favorables, se evidenció una tendencia hacia el manejo de las normas y costumbres para el ejercicio de la dirección, lo que se corresponde con la presencia del estilo laissez faire, en una porción poco significativa. Esto se considera una expresión conductual que una parte minoritaria expresa como una devaluación de los aspectos humanos, y una tendencia al burocratismo, percepción que no es significativa, pero que puede convertirse en un elemento disonante.

En relación con la responsabilidad directiva, se presenta una polarización entre el trabajo en equipo y el trabajo por delegación en el individuo. Adicionalmente, una presencia significativa de las responsabilidades directivas asignadas por control normativo, lo cual implica que no hace mayoritario el trabajo en equipo, como debería corresponderse a una situación de transición, de altas exigencias y apoyo colectivo intergrupal.

En la toma de decisiones, se presenta un equilibrio entre las de carácter institucional-normativo y la casuística-coyuntural, lo cual es un factor altamente positivo, por cuanto el personal gerencial rechaza el método personal y espontáneo, de la intuición y la experiencia para la toma de decisión y se centra en los objetivos y metas de la organización y en el análisis de la realidad.

Esta percepción de los gerentes está influida por la importancia que ha adquirido el trabajo en equipo que se practica en distintas instancias, el importante rol que juega la preparación de los cuadros directivos y los mecanismos internos de evaluación y control de la gestión. Estos tres factores han conducido a asumir mayoritariamente mecanismos institucionales y a la búsqueda del consenso que se viene fortaleciendo en la empresa, luego que se reafirmo en su carácter nacionalista.

En cuanto a la asignación de tareas, se presenta una disyuntiva entre la delegación que tiende a valorar a la persona y la interdependencia y responsabilidad compartida, que tiende a valorar al 
Cultura Organizacional y Gestión de la Calidad en una Empresa del Estado venezolano Salcedo, Irma y Romero, Juan José

grupo; estando este estilo más arraigado que el primero, esto resultó un dato interesante en las percepciones emitidas, ya que presenta una ligera diferencia con la forma como asumen la responsabilidad directiva.

Aquí juega un papel muy importante la división del trabajo que se desarrolla en la empresa. Esta división del trabajo está relacionada con la elevada complejidad del proceso tecnológico que requiere un equilibrio entre el trabajo en grupo y la responsabilidad individual

El manejo de conflictos, se ubica en su totalidad, hacia la aplicación del método de negociación y solución de problemas, lo cual se constituye en un factor altamente favorable para el ejercicio de una cultura de participación, que se corresponde con la implantación de un sistema que busca la calidad centrada en la eficiencia, eficacia y efectividad de las acciones humanas.

Este resultado tiene que ver con; el fortalecimiento de un clima organizacional que valora los compromisos mutuos entre el supervisor y los supervisados, la instauración de un sistema de reconocimientos del desempeño y el respeto de la dignidad de todos los trabajadores.

El abordaje de los valores, principios y antivalores de la cultura organizacional, nos permitió caracterizar los factores subjetivos que motorizan la conducta de los gerentes en la empresa investigada. Como resultado de ello, esta investigación aporta una escala jerarquizada en función del nivel de aceptación que expresaron los entrevistados en torno a la incidencia de éstos en su comportamiento organizacional, lo que resulta de gran utilidad para la consolidación de una cultura de la empresa.

En relación a los valores, se identificaron como los cinco más importantes: la responsabilidad, la honestidad, el respeto, la disciplina y la motivación al logro. En relación a los principios se aporta una escala jerárquica, donde se destacan como los cinco más importantes: el liderazgo, la comunicación, el mejoramiento continuo, la cooperación y la capacitación. En cuanto a los antivalores, la escala presenta un nivel de rechazo hacia las siguientes desviaciones de la conducta gerencial: la deshonestidad, la mentira, la injusticia, la agresividad y la envidia.

Este hallazgo permite caracterizar las representaciones conductuales y motivacionales de la acción gerencial en la empresa investigada y deberá ser tomada muy en cuenta para avanzar en la estrategia de cambio que hoy vive esa organización. Por lo que se puede interpretar que existen condiciones altamente favorables para la implantación de un sistema de gestión de la calidad, centrada en el alto valor del personal que la dirige.

\section{Conclusiones y recomendaciones}

La necesidad de incorporar este nuevo sistema de gestión, surgió de un análisis estratégico básico, donde se consideraron algunas macrovariables de la planificación global, las cuales están referidas a las dimensiones económicas, sociales y políticas que intervienen en el entorno de esta empresa del Estado, y que de alguna manera condicionan su comportamiento organizacional. 
La información aportada por todos los gerentes de la empresa investigada, evidencia que se ha conceptualizado adecuadamente la calidad, considerándosele como una variable inmanente de la acción gerencial. Por ello, los directivos de la empresa, previendo la próxima implantación del sistema de gestión de la calidad, están creando un clima de participación, donde las decisiones se distribuyan equitativamente en toda la organización, con procesos comunicacionales dirigidos de manera ascendente, descendente y lateral. Se está conformando un escenario favorable, para que los trabajadores, tanto directivos como supervisados, alcancen un nivel de motivación adecuado para lograr la calidad, a través de sus tres indicadores básicos, como son: La eficiencia, concebida en términos de la utilización optima de los recursos para el cumplimiento de las metas. La eficacia que se refiere a la capacidad para lograr los objetivos y metas planificadas por la organización, y. La efectividad que se expresa a través del impacto de los proyectos de la empresa en el entorno.

Es decir, como un signo altamente positivo, ha comenzado a surgir la cultura de la calidad, identificándose como una ventaja comparativa, asociada al cambio tecnológico y al fortalecimiento de los recursos humanos. La gestión empresarial se ha formulado como un reto para la aplicación de un modelo creativo, que conlleva a enfrentar las exigencias de un medio ambiente muy cambiante, ante el cual la empresa se ha venido desempeñando con éxito, tanto en la elevación de la efectividad y en la investigación y aplicación de este nuevo enfoque de planificación, como también el ejercicio de las nuevas teorías gerenciales.

En este proceso de transformación gerencial, ha jugado un papel muy importante el entrenamiento, actualización y desarrollo desplegados hacia el personal ubicado en todos los niveles de la organización, convirtiéndose en el motor del proceso. En este sentido, la empresa se está fortaleciendo para afrontar la complejidad del entorno de competencia, con clientes exigentes, y con fuertes exigencias ambientales, de higiene y seguridad laboral. Todo ello acompañado de una visión nacionalista, que refirma a esta organización como empresa pública del Estado venezolano, ya que esta empresa estuvo sometida durante un reciente periodo, a un alto nivel de incertidumbre con la pretendida imposición de un esquema de privatización, que a pesar de que resultó fallido, afectó circunstancialmente su nivel de identidad, como una organización fundamental para la estrategia del desarrollo endógeno sustentable.

Entre los factores que se verán fortalecidos con la aplicación del sistema de gestión de la calidad, están: El trabajo en equipo, el fortalecimiento de los principios aprender haciendo y aprender a aprender, la integración y aplanamiento de la estructura, a través de la reducción de los niveles jerárquicos y el mejoramiento de la comunicación interna.

En este marco de modificación cualitativa, la función de personal ha pasado a ser determinante, en el sentido de su alta responsabilidad con el desarrollo de un proceso educativo, para que la gente comprenda que la cultura organizacional es una de las más importantes venta- 
Cultura Organizacional y Gestión de la Calidad en una Empresa del Estado venezolano Salcedo, Irma y Romero, Juan José

jas competitivas de la gestión empresarial. En este sentido, se les recomienda el desarrollo de un programa de formación con todos los trabajadores que conforman el colectivo laboral, con la finalidad de que conozcan los valores y principios de la cultura organizacional de la empresa y los proyecten permanentemente hacia un desempeño laboral de excelencia. Por otra, se recomienda que este programa rechace en primer lugar, el impacto negativo de los factores desfavorables de la cultura como son los antivalores, los cuales afectan a los trabajadores y en general a la cultura de la calidad, por la cual se ha orientando la filosofía de gestión.

Finalmente se considera conveniente incorporar, un perfil gerencial, basado en: el compromiso social, la visión compartida y el respeto por el cliente.

El impulso de los valores y principios contemplados en el sistema de gestión de la calidad deben orientarse para mejorar el sentido de pertenencia y alcanzar una conducta deseada que guíe el desempeño de cada uno de los miembros de la empresa.

\section{Referencias Bibliográficas}

Cantú D, Humberto (2001). Desarrollo de una Cultura de Calidad. México. Editorial Mc Graw Hill.

Cruz, Teresa (2001). La cultura organizacional. La Habana. Ediciones CEEC $\mathrm{UH}$.

Davis, Keith y Newstrom, John (2000). Comportamiento Humano en el Trabajo. México. Editorial Mc Graw Hill.
Ferrater M., José (1974). Diccionario de Filosofía. Buenos Aires. Editorial Sudamericana.

FONDONORMA (2000). Norma ISO 9001. Caracas. Ediciones fondonorma.

Granell, Elena y Garaway, David (1997). Éxito Gerencial y Cultura. Retos y oportunidades en Venezuela. Venezuela. Ediciones IESA.

Guédez, Víctor (1996). Gerencia, Cultura y Educación. Caracas. Editorial Tropykos.

IVEPLAN (1991). Planificación y Política. Caracas. Editado por Instituto Venezolano de Planificación.

IVEPLAN (1993). Planificación y Gestión: De la Teoría al Método. Caracas. Editado por el Instituto Venezolano de Planificación.

Krause, Donald (1998). El Camino del Líder. Buenos Aires. Madrid. Editorial EDAF.

Montero, Maritza (1999). Ideología, alineación e identidad nacional. Caracas. Ediciones UCV.

Ostos, Raiza (2000). Filosofía de Gestión, Ética y Valores. Caracas. Edita R.O, Consultora Gerencial.

Ouchi, William (1982). Teoría Z. Editorial Fondo Educativo Interamericano, S.A. México.

Pérez, Carlota (1998). Desafíos sociales y políticos del cambio de paradigma tecnológico. Caracas. Editado por UCAB.

Robbins, Stephen (1991). Comportamiento Organizacional. México. Editorial Prentice-Hall.

Romero García, O. (1996). Memorias EVEMO 6. Mérida. Edita ROGYA.

Schein. Edgar H. (1988). La Cultura Empresarial y Liderazgo. Barcelona. Edita Plaza \& Janes. 